\title{
An alternatively efficient method (DBEM) for simulating the electrostatic field and levitating force of a MEMS combdrive
}

\author{
Yunn-Shiuan Liao ${ }^{1}$, Shiang-Woei Chyuan ${ }^{1}$ and Jeng-Tzong Chen ${ }^{2}$ \\ ${ }^{1}$ Department of Mechanical Engineering, National Taiwan University, Taipei, Taiwan, \\ Republic of China \\ ${ }^{2}$ Department of Harbor and River Engineering, National Taiwan Ocean University, Keelung, \\ Taiwan, Republic of China \\ E-mail: yeaing@iris.seed.net.tw
}

Received 1 March 2004, in final form 28 April 2004

Published 21 June 2004

Online at stacks.iop.org/JMM/14/1258

doi:10.1088/0960-1317/14/8/020

\begin{abstract}
For MEMS combdrive design, the reduction of levitating force due to electrostatic fields is very important, and an accurate electrostatic analysis is essential and indispensable. For diverse MEMS combdrive designs, the boundary element method (BEM) has become a better method than the domain-type finite element method (FEM) because the BEM can provide a complete solution in terms of boundary values only, with substantial saving in modeling effort. Since dual BEM (DBEM) has some advantages over conventional BEM for a singularity, the DBEM was used to simulate the fringing of field around the edges of the fixed fingers and movable fingers of MEMS combdrives for diverse design cases. A number of electrostatic problems for typical MEMS combdrive designs were analyzed to check the efficiency and validity of this new technique. It is found that the numerical results computed by coarse mesh DBEM match the reference data from a large refined mesh FEM very well, and the accuracy and performance of DBEM are also better than those of conventional BEM for solving the electric intensity field of MEMS combdrives. By way of the DBEM presented in this paper, an accurate and reasonable electrostatic field can be obtained, and the follow-up control method of levitating force for the MEMS combdrive can be implemented more precisely.
\end{abstract}

(Some figures in this article are in colour only in the electronic version)

\section{Introduction}

A MEMS (microelectromechanical systems) combdrive as shown in figure 1 usually has two sets of fingers. The one that is connected to the substrate, is called the fixed fingers (stationary electrode), and the other, which is released from the substrate, is called the movable fingers. When two different voltages are applied to these two sets of fingers, the resulting electrostatic force drives the movable fingers towards the fixed ones. Thus, motion is produced by this combdrive in the direction of the movement of the movable fingers [1]. Because a combdrive can be designed for either an electrostatic actuator or capacitive sensing, it has become a very important device in MEMS [2]. Basically, in-plane interdigitated combdrives are used in in-plane or small out-of-plane/torsional motions [3], and asymmetric combdrives can be utilized to generate large out-of-plane or torsional motions [4]. Generally speaking, in a typical in-plane interdigitated combdrive, the capacitance is linear with displacement, resulting in an electrostatic driving force, which is independent of the position of the movable fingers except at the ends of the range of travel [5]. But, for some special applications, combdrives with variable-gap profiles can be designed, which will deliver the desired driving 


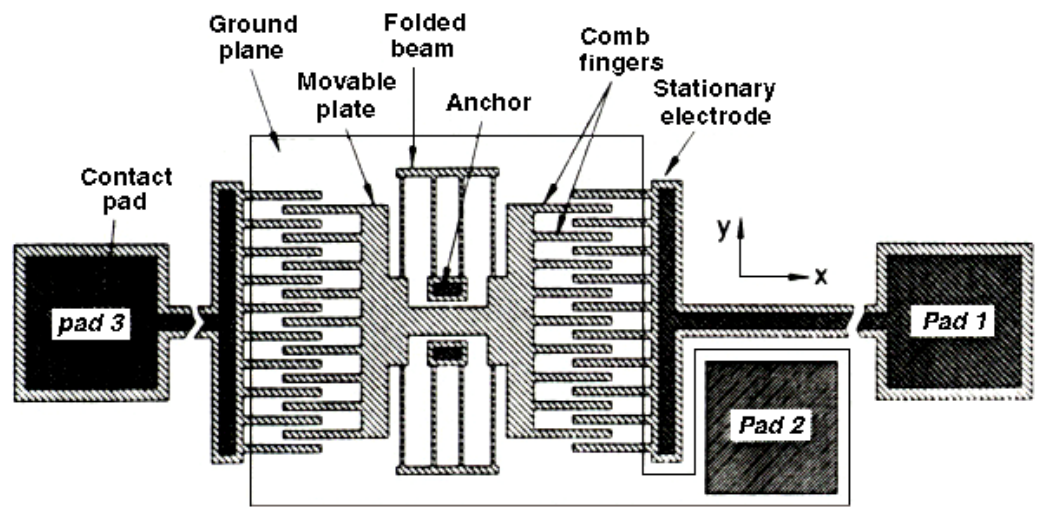

Figure 1. Layout of a linear lateral resonator driven and sensed with interdigitated capacitors (electrostatic combdrive).

force profiles by solving an appropriate inverse problem [6].

For in-plane interdigitated combdrives, some advantages [2] could be found: (1) vibrational amplitudes over $10 \mu \mathrm{m}$ are possible with relatively high quality factors at atmospheric pressure, in contrast to structures which move normal to the surface of the substrate. (2) The combdrive capacitance is linear with displacement, resulting in an electrostatic drive force, which is independent of vibrational amplitude. (3) Flexibility in the design of the suspension for the resonator. Though surface-micromachined polysilicon resonators, which are driven by interdigitated capacitors, have several aforementioned attractive properties, it is essential that both the movable fingers and the fixed fingers of combdrives remain coplanar for high quality MEMS devices. It was reported that $2 \mu \mathrm{m}$ thick polysilicon resonators with compliant folded-beam suspensions have been observed to levitate over $2 \mu \mathrm{m}$ when driven by an electrostatic comb biased with a dc voltage of $30 \mathrm{~V}$ [2]. Because the levitation effect will seriously downgrade the performance and reliability of MEMS devices, how to obtain the actual electrostatic force responsible for levitation plays a very important role. Since the calculation of surface charge density around the movable fingers is the base for calculating the levitating force, knowledge of the electric potential $V$ and electric field intensity $E$ around the fixed fingers, movable fingers and ground plane of MEMS combdrives, considering the fringing of field around the edge, is needed first for engineering design. Searching for an efficient method for the electrostatic analysis task is very important for engineers.

The paper is organized as follows. The numerical methods for MEMS and EM devices are introduced in section 2. Section 3 involves the reason for choosing DEBM for MEMS combdrives, and some comparisons between the dual BEM (DBEM) and conventional boundary element methods (BEM) are described. In section 4, we introduce the procedure of dual integral equations for electrostatic problems. Before the DBEM was used to solve and simulate real MEMS devices and structures, a benchmark test was performed against the analytical solution to compare its computational accuracy (section 5). In section 6, the computational results of DBEM simulation for the MEMS combdrive levitation are provided and investigated, and the numerical accuracy and performance of DBEM and conventional BEM are also compared. Some remarks based on the reported results are discussed in section 7 . Finally, there is a concise conclusion in section 8 .

\section{Numerical methods for MEMS and EM devices}

Basically, electrical engineers are familiar with electrostatic problems, and diverse numerical methods have been regularly used in MEMS and EM (electromagnetics) [7]. Among different numerical approaches, the finite element method (FEM), which is based on the representation and approximate solution of boundary value problems of engineering mathematics in terms of partial differential equations $[8,9]$, and the boundary element method based on integral equations [10] have moved from being research tools for scientists to become powerful design tools for engineers. One of the main advantages of the BEM, when compared to the FEM, is that discretizations are restricted only to the boundaries, making data generation much easier. The BEM is also ideally suited to the analysis of external problems where domains extend to infinity, since discretizations are confined to the internal boundaries with no need to truncate the domain at a finite distance and impose artificial boundary conditions, and to problems involving some form of discontinuity or singularity, due to the use of singular fundamental solutions as test functions. It is also interesting to point out that the unknowns in the BEM are a mixture of the potential and its normal derivative, rather than the potential only as in the FEM. This is a consequence of the BEM being a 'mixed' formulation, and constitutes an important advantage over FEM. Especially for the diverse gaps of MEMS combdrives-gaps between fingers and from fingers to the ground plane-many laborious works of finite element modeling are needed, compared to those of the boundary element model, because the BEM can provide a complete solution in terms of boundary values only, with substantial saving in modeling effort. Therefore, there is no doubt that the BEM has become a very appealing approach in numerical simulation of MEMS [11].

As we know, with the rapid increase in device density and working frequency in VLSI circuits, the electrical characteristics of interconnects are becoming more important factors governing the circuit performances such as delay, power consumption, reliability, etc. This has increased the interest in efficient methods for calculating electrical parameters of interconnects. Therefore, many researchers 
have used diverse accelerated BEMs (e.g. FastCap, GIMEI, ODDM, QMM, etc) for efficiently solving the large-scale problem of VLSI \& EM systems [12-16]. To the best knowledge of the authors, accelerated BEMs are very efficient for large-scale problems because they can reduce $\mathrm{CPU}$ time and memory requirement from exponential order to logarithmic order. For a problem with $N$ degrees of freedom, or $N$ unknowns, the accelerated BEMs use $\mathrm{O}(N \log N)$ memory, and close to $\mathrm{O}(N \log N)$ time, as opposed to the traditional methods requiring $O\left(N^{2}\right)$ memory and $O\left(N^{2}\right)$ time [17]. As $N$ becomes very large, there will be a tremendous disparity in memory and time usage between accelerated BEMs and conventional BEMs. Although many BEM researchers have used diverse accelerated BEMs for large-scale problems (e.g. complex VLSL modeling) they need to deal with the huge dense matrix from the BEM. But, for the concerned levitation problem of MEMS combdrives, it is not necessary to set up a huge BEM model because only the surface charge distribution around the boundary of movable fingers is needed. In reality, the accelerated BEM may have an advantage over DBEM for large-scale problems, but this predominance is not obvious for non-large-scale problems such as the cases presented in this paper because the model of a MEMS combdrive is not as large as a VLSI system. As the accelerated BEM cannot display its advantage (computational efficiency) over the DBEM for non-large-scale problems, the DBEM was used in this paper because it is an alternate efficient method for MEMS combdrives.

Although some simplified numerical models for electrostatic combdrives can be found in $[4,5]$, there are still three types of fringing fields not taken into account, which result from the ground plane, widths and heights of fixed and movable fingers. In order to obtain more reasonable computational results for the electric field, the DBEM is employed and developed to analyze electrostatic problems for MEMS combdrive levitation, considering the fringing of field around the edges, in the paper. After using the DBEM to accurately calculate the electrostatic response of a comb finger biased with a dc voltage, the induced vertical force per unit length of the movable comb finger at different levitation positions can be obtained. Then this vertical force density can be plotted against levitation at different dc bias voltages, as in $[4,5]$. In order to check the validity of the numerical model presented in this paper, several examples of different inplane interdigitated combdrive designs from [3] are furnished, and the solutions of the DBEM are compared with analytical solutions if available and with an FEM package [18].

\section{The reason for choosing DBEM for MEMS devices}

As we all know, there are two commonly used electrostatic actuators for linear motion: combdrive and parallel-plate actuators. For a thin parallel-plate capacitor, a degenerate boundary will be formed while using the conventional BEM. (The degenerate boundary refers to a boundary, two portions of which approach each other such that the exterior region between the two portions becomes infinitely thin [19].) The DBEM can deal with this singularity very efficiently and directly, but the conventional BEM cannot work well without an artificial boundary technique for the interface since the coincidence of the boundaries gives rise to an ill-conditioned problem. The sub-domain technique in the conventional BEM with artificial boundaries for a degenerate boundary has been introduced to ensure a unique solution. The main drawback of the technique is that the deployment of artificial boundaries is arbitrary and, thus, cannot be implemented easily into an automatic procedure. In addition, model creation is more troublesome than in the single domain approach. To tackle such degenerate boundary electrostatic problems the DBEM has been proposed in [20], and all the abovementioned boundary value problems can be solved efficiently in the original single domain if using the DBEM. Besides the degenerate boundary problem, there are also several advantages (e.g. the degenerate scale rank deficient problem, corner problem, the calculation of the tangent flux on and near the boundary, the adaptive boundary element methods, etc) for the DBEM over the conventional BEM, which can be found in some published papers.

\subsection{Degenerate scale (rank deficient) problem}

Besides degenerate boundary, degenerate scale is another important degenerate source for singularity of the BEM. For problems with special scale of geometric shape, many researchers have found that the influence matrix of the weakly singular kernel may be singular for the Dirichlet problem $[21,22]$. Therefore, if encountering electrostatic problems with some specific geometry, the singularity caused by a degenerate scale will be encountered, since the influence matrix is rank deficient, and BEM numerical results become unstable. From some published papers [23, 24], we can find that the numerical difficulty of degenerate scale concerning the conventional BEM can be solved by way of the hypersingular formulation instead of using the singular formulation in the DBEM.

\subsection{Corner problem}

The corner problem with Dirichlet boundary condition is another problem in which the number of equations is not sufficient for the conventional BEM. Again, the hypersingular integral formulation of the DBEM plays a role in providing independent constraints for the boundary unknowns. Unfortunately, the singular equations of the BEM alone cannot distinguish the normal vectors of the collocation points at the corner. The hypersingular integral formulation of DBEM can be collocated to the points before the corner and after the corner with two different independent normal vectors, causing the equations to be independent, as shown in [25] for the twodimensional Laplace equation.

\subsection{Calculation of the tangent flux on and near the boundary}

Because some BEM researchers have shown that the accuracy of the numerical derivate using the BEM is lower than that of the direct calculation of the boundary stress using the hypersingular formulation of the DBEM, since the integral representation of the solution exhibits the jump behavior across the boundary, the stress or flux near the boundary often displays the Gibbs phenomenon [26]. Therefore, the hypersingular integral equation of DBEM can be used to directly calculate the tangent electric field instead of using the numerical derivative 
of the obtained potential field. The tangent derivative along the boundary has been formulated in terms of both the boundary potential and the boundary normal flux. Of course, the numerical error from the conventional BEM facing the fringing of field around the edges could be eliminated.

\subsection{Adaptive boundary element methods}

An essential ingredient for all adaptive BEMs is a reliable estimate of the local error. The hypersingular integral equation used in the DBEM is a complementary equation available for error estimation. Using this concept, the error indicator can successfully track the form of the exact error curve [27].

\section{Dual integration equations for electrostatic problems}

For a homogeneous medium, the governing equation of electrostatics can be written in $\nabla^{2} V=-\rho / \varepsilon$, where $\nabla^{2}$ is the Laplacian operator. This Poisson's equation states that the divergence of the gradient of electric potential $(V)$ equals $-\rho / \varepsilon$ for a simple medium, where $\varepsilon$ is the permittivity of the medium and $\rho$ is the volume density of free charges [28]. At points in a simple medium where there is no free charge, the Poisson equation is reduced to $\nabla^{2} V=0$, which is known as Laplace's equation. The Laplace equation plays a very important role in MEMS and EM. It is the governing equation for electrostatic problems involving a set of conductors, such as capacitors, maintained at different potentials. Once $V$ is found from Laplace's equation, $E$ (electric field intensity) can be determined from $-\nabla V$, and the charge distribution on the conductor surfaces can be determined from $\rho_{\mathrm{s}}=\varepsilon E n$.

As we know, the electrostatic levitating forces acting on the movable fingers need to be obtained first before solving the mechanical response of MEMS combdrives; hence, the electrostatic analysis work to obtain the distribution of surface charge density around the movable fingers is the base for calculating the levitating force density. Therefore, choosing an efficient method such as the DBEM is necessary for simulating the exterior electric field. The electrostatic problem consists of finding the unknown potential function $\Phi$ (or V) in the partial differential equation. In addition to the fact that $\Phi$ satisfies the Laplace equation within a prescribed solution region $D$, the potential function $\Phi$ must satisfy certain conditions on $B$ which is the boundary of $D$. Usually these boundary conditions are the Dirichlet $(\Phi(x)=f(x))$ and Neumann $\left(\partial \Phi(x) / \partial n_{x}=g(x)\right)$ types, where $f(x)$ and $g(x)$ denote known boundary data, and $n_{x}$ is the unit outer normal vector at the point $x$ on the boundary $B$ (see figure 2 ). Therefore, the governing equation and boundary conditions of electrostatic problems could be written in the following form.

Governing equation:

$$
\nabla^{2} \Phi(x)=0, \quad x \text { in } D .
$$

For the interior electrostatic problems, the dual integral equations were derived as follows:

$$
\begin{gathered}
\pi \Phi(x)=\mathrm{CPV} \int_{B} T^{i}(s, x) \Phi(s) \mathrm{d} B(s) \\
-\operatorname{RPV} \int_{B} U^{i}(s, x)\left[\partial \Phi(s) / \partial n_{s}\right] \mathrm{d} B(s)
\end{gathered}
$$

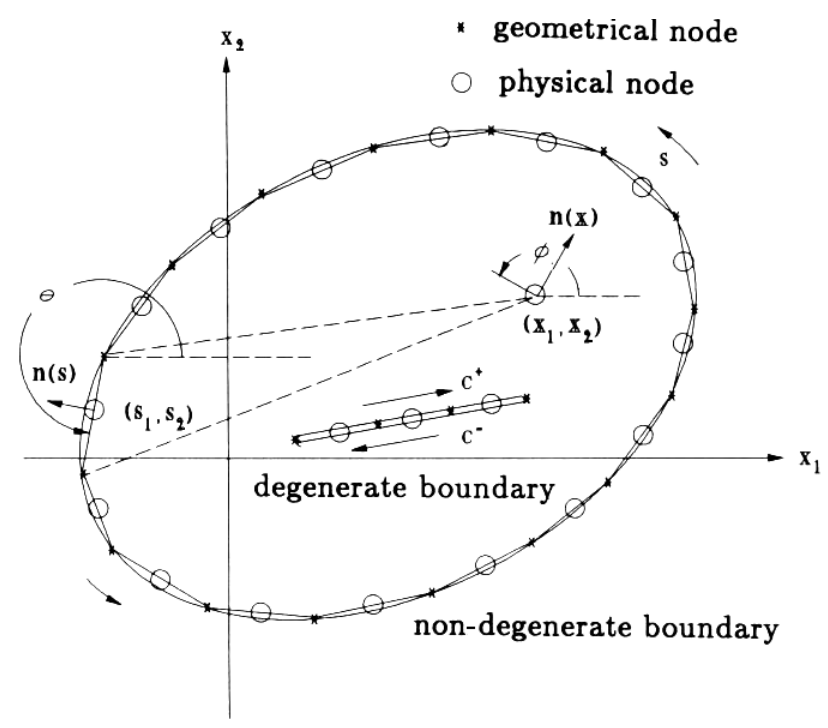

Figure 2. Boundary element discretization for degenerate boundary and non-degenerate boundary.

$$
\begin{gathered}
\pi\left[\partial \Phi(x) / \partial n_{x}\right]=\mathrm{HPV} \int_{B} M^{i}(s, x) \Phi(s) \mathrm{d} B(s) \\
-\mathrm{CPV} \int_{B} L^{i}(s, x)\left[\partial \Phi(s) / \partial n_{s}\right] \mathrm{d} B(s)
\end{gathered}
$$

where $x$ is on the boundary $B, \Phi(x)$ and $\left[\partial \Phi(x) / \partial n_{x}\right]$ are the potential and flux, $U^{i}, T^{i}, L^{i}$ and $M^{i}$ are the four kernels $\left(U(s, x)=\ln (r) ; T(s, x)=\partial U(s, x) / \partial n_{s} ; L(s, x)=\right.$ $\partial U(s, x) / \partial n_{x} ; M(s, x)=\partial^{2} U(s, x) / \partial n_{x} \partial n_{s}$, where $r=$ $|s-x|, s$ and $x$ being the position vectors of the points $s$ and $x$, respectively, and $n_{s}$ is the unit outer normal vector at point $s$ on the boundary) in the dual integral equations for the interior problems, and RPV, CPV and HPV denote the Riemann principal value, Cauchy principal value and Hadamard principal value, respectively [19, 20]. Generally, equation (2) is called the singular boundary integral equation, and equation (3) is called the hypersingular boundary integral equation. Since the hypersingular boundary integral equation plays an important role in the degenerate problems, many researchers have paid much attention to this. Extending equations (2) and (3) of the interior electrostatic problem to the exterior problem by considering the regularity condition at infinity for the integral on the infinite boundary $B_{\infty}$, we have

$$
\begin{gathered}
\pi \Phi(x)=\operatorname{CPV} \int_{B} T^{\mathrm{e}}(s, x) \Phi(s) \mathrm{d} B(s) \\
-\operatorname{RPV} \int_{B} U^{\mathrm{e}}(s, x)\left[\partial \Phi(s) / \partial n_{s}\right] \mathrm{d} B(s) \\
\pi\left[\partial \Phi(x) / \partial n_{x}\right]=\operatorname{HPV} \int_{B} M^{\mathrm{e}}(s, x) \Phi(s) \mathrm{d} B(s) \\
-\operatorname{CPV} \int_{B} L^{\mathrm{e}}(s, x)\left[\partial \Phi(s) / \partial n_{s}\right] \mathrm{d} B(s)
\end{gathered}
$$

where the superscript ' $\mathrm{e}$ ' denotes the exterior problem.

The commutativity property of the trace operator and the normal derivative operator provides us with alternative ways to calculate the Hadamard principal value analytically [19]. First, L'Hospital's rule is employed in the limiting process. Second, the normal derivative of the Cauchy principal value 


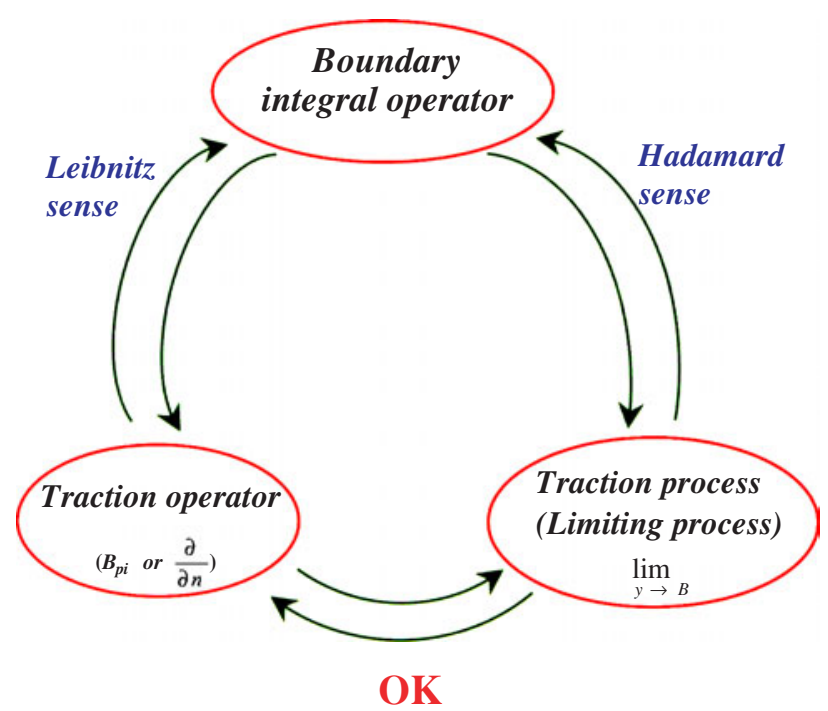

Figure 3. Commutativity of trace and differential operator.

should be taken carefully by using Leibnitz' rule, and then the finite part can be obtained. The finite part has been termed the Hadamard principal value or Mangler's principal value. In the derivation of dual equations, two alternatives can be applied to determine the Hadamard principal value as shown in figure 3.

\subsection{Trace operator first and differential operator second}

$$
\begin{aligned}
\operatorname{HPV} & \int_{B} M(s, x) u(s) \mathrm{d} B(s) \\
& =\frac{\partial}{\partial n_{x}}\left\{\mathrm{CPV} \int_{B} T(s, x) u(s) \mathrm{d} B(s)\right\} .
\end{aligned}
$$

For simplicity, a constant element is adopted, i.e. $u(s)=1$, and equation (6) reduces to

$$
\begin{aligned}
\frac{\mathrm{d}}{\mathrm{d} x} & \left\{\operatorname{CPV} \int_{a}^{c} \frac{-1}{(x-s)} \mathrm{d} s\right\} \\
& =\frac{\mathrm{d}}{\mathrm{d} x}\left\{\int_{a}^{x-\varepsilon} \frac{-1}{(x-s)} \mathrm{d} s+\int_{x+\varepsilon}^{c} \frac{-1}{(x-s)} \mathrm{d} s\right\} \\
& =\frac{1}{a-x}-\frac{1}{c-x}
\end{aligned}
$$

after using the Leibnitz rule.

\subsection{The differential operator first and trace operator second}

$\operatorname{HPV} \int_{B} M(s, x) u(s) \mathrm{d} B(s)=\lim _{y \rightarrow x} \int_{B} M(s, y) u(s) \mathrm{d} B(s)$.

Similarly, the constant element scheme can simplify equation (7) into

$$
\begin{aligned}
\lim _{y \rightarrow 0} & \int_{a}^{c} \frac{1}{(x-s)^{2}+y^{2}} \mathrm{~d} s \\
& =\lim _{y \rightarrow 0} \frac{1}{y}\left[\tan ^{-1}\left(\frac{y}{a-x}\right)-\tan ^{-1}\left(\frac{y}{c-x}\right)\right] \\
& =\frac{1}{a-x}-\frac{1}{c-x}
\end{aligned}
$$

after using

$$
\lim _{\alpha \rightarrow 0} \frac{\tan ^{-1} \alpha}{\alpha}=1
$$

and

$$
\tan ^{-1} \alpha+\tan ^{-1} \frac{1}{\alpha}=\frac{\pi}{2} .
$$

The linear algebraic equations for an interior electrostatic problem discretized from the dual boundary integral equations can be written as

$$
\begin{aligned}
& {\left[T_{p q}^{i}\right]\left\{\Phi_{q}\right\}=\left[U_{p q}^{i}\right]\{\partial \Phi / \partial n\}_{q}} \\
& {\left[M_{p q}^{i}\right]\left\{\Phi_{q}\right\}=\left[L_{p q}^{i}\right]\{\partial \Phi / \partial n\}_{q}}
\end{aligned}
$$

where $\left\{\Phi_{q}\right\}$ and $\{\partial \Phi / \partial n\}_{q}$ are the boundary potential and flux, and the subscripts $p$ and $q$ correspond to the labels of the collocation point and integration element, respectively. For the exterior electrostatic problem, we have

$$
\begin{aligned}
{\left[T_{p q}^{\mathrm{e}}\right]\left\{\Phi_{q}\right\} } & =\left[U_{p q}^{\mathrm{e}}\right]\{\partial \Phi / \partial n\}_{q} \\
{\left[M_{p q}^{\mathrm{e}}\right]\left\{\Phi_{q}\right\} } & =\left[L_{p q}^{\mathrm{e}}\right]\{\partial \Phi / \partial n\}_{q} .
\end{aligned}
$$

The influence coefficients of the four square matrices $[U],[T],[L]$ and $[M]$ can be represented as

$$
\begin{gathered}
U_{p q}=\mathrm{RPV} \int_{B_{q}} U\left(s_{q}, x_{p}\right) \mathrm{d} B\left(s_{q}\right) \\
T_{p q}=-\pi \delta_{p q}+\mathrm{CPV} \int_{B_{q}} T\left(s_{q}, x_{p}\right) \mathrm{d} B\left(s_{q}\right) \\
L_{p q}=\pi \delta_{p q}+\mathrm{CPV} \int_{B_{q}} L\left(s_{q}, x_{p}\right) \mathrm{d} B\left(s_{q}\right) \\
M_{p q}=\operatorname{HPV} \int_{B_{q}} M\left(s_{q}, x_{p}\right) \mathrm{d} B\left(s_{q}\right)
\end{gathered}
$$

where $B_{q}$ denotes the $q$ th element and $\delta_{p q}=1$ if $p=q$, otherwise it is zero. The explicit form will be derived in the following section. According to the dependence of the out-normal vectors in these four kernel functions for the interior and exterior electrostatic problems, their relationship can easily be found:

$U_{p q}^{i}=U_{p q}^{\mathrm{e}}$

$M_{p q}^{i}=M_{p q}^{\mathrm{e}}$

$T_{p q}^{i}=-T_{p q}^{\mathrm{e}}$

if $p \neq q ; \quad T_{p q}^{i}=T_{p q}^{\mathrm{e}}$

if $p=q$

$L_{p q}^{i}=-L_{p q}^{\mathrm{e}}$

if $p \neq q ; \quad L_{p q}^{i}=L_{p q}^{\mathrm{e}}$

if $p=q$.

\section{An example of benchmark test for DBEM}

Before the DBEM was used to solve and simulate real MEMS devices and structures, the following benchmark test was performed against the analytical solution to verify its computational accuracy.

Benchmark test. Two grounded, semi-infinite, parallel-plane electrodes are separated by a distance $b$. A third electrode perpendicular to and insulated from both is maintained at a constant potential $V_{0}$ (see figure 4). Determine the electric potential distribution in the region enclosed by the electrodes. 


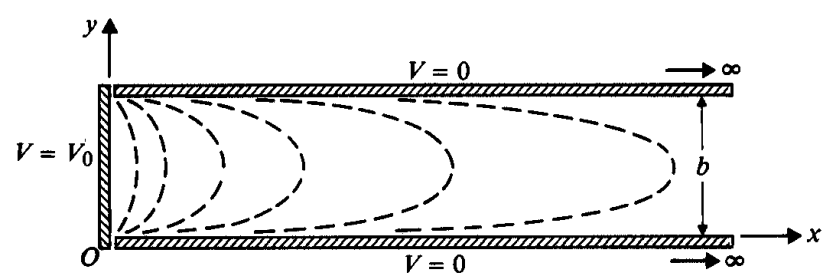

Figure 4. Cross-sectional figure for benchmark test. The plane electrodes are infinite in the $z$-direction.

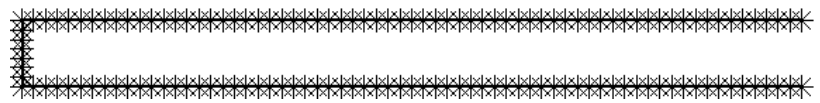

Figure 5. The related DBEM mesh discretization of benchmark test (146 elements and 146 nodes).

\subsection{Analytical solution}

Using the method of separation of variables, the desired electric potential distribution $V(x, y)$ could be obtained [28],

$$
V(x, y)=\Sigma C_{n} \exp (-n \pi x / b) \sin (n \pi y / b)
$$

where $C_{n}=\left(4 V_{0} / n \pi\right)$ if $n$ is odd; $C_{n}=0$ if $n$ is even; $n=1,3,5, \ldots, \infty$. Equation (20) is a rather complicated expression to plot; but since the amplitude of the sine terms in the series decreases very rapidly as $n$ increases, only the first few terms are needed to obtain a good approximation. Several equipotential lines are sketched in figure 4.

\subsection{DBEM analysis}

For convenience, the value of $b$ is assumed to be 3.333 , and four points will be analyzed using rough mesh discretization (146 elements and 146 nodes, see figure 5) of the DBEM, and compared with the analytical data computed from equation (20). The results of electric potential under the DBEM and analytical methods are listed in table 1. Because the errors between the analytical method and the DBEM are lower than $1 \%$, the simulation data of the DBEM presented in this paper are valid and convincing for electrical engineers.

\section{DBEM simulation for MEMS combdrive levitation}

The successful electrostatic actuation of micromechanical structures requires a ground plane under the structure in order to shield it from relatively large vertical fields [3]. In order to demonstrate the suitability of DBEM, several electrostatic combdrive problems under different designs proposed by Tang et al [3] were used. In this paper, a $4 \mu \mathrm{m}$ wide $\times 2 \mu \mathrm{m}$ thick comb finger excited by two identically sized electrodes situated $2 \mu \mathrm{m}$ away from both sides of the finger, and $2 \mu \mathrm{m}$ above a grounded substrate was used (see figure 1).

Case 1. A comb finger under levitating force (normal to the substrate) induced by two adjacent electrodes biased at a positive potential $V_{p}$ is shown in figure 6. Let us determine the electric field distribution.

From figure 6 , one can see that there is an obvious fringing of field around the edges of fixed and movable fingers, and the physical behavior (e.g. electric potential and

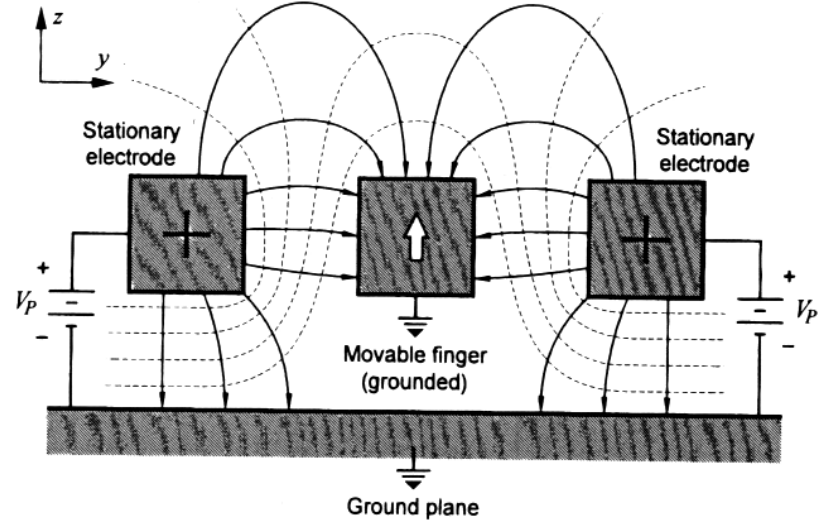

Figure 6. Cross section of the potential contours (dashed lines) and the electric fields (solid lines) of a comb finger under levitating force induced by two adjacent electrodes biased at a positive potential.

Table 1. The results of electric potential of benchmark test under DBEM and analytical methods.

\begin{tabular}{llll}
\hline $\begin{array}{l}\text { Locations } \\
(x, y)\end{array}$ & $\begin{array}{l}V(x, y) \text { from } \\
\text { DBEM }\end{array}$ & $\begin{array}{l}V(x, y) \text { from } \\
\text { analytical method }\end{array}$ & Error \\
\hline$(0.667,2.778)$ & $0.40500 V_{0}$ & $0.407890 V_{0}$ & $-0.71 \%$ \\
$(3.333,0.556)$ & $0.027363 V_{0}$ & $0.027536 V_{0}$ & $-0.63 \%$ \\
$(6.667,1.111)$ & $0.002043 V_{0}$ & $0.002058 V_{0}$ & $-0.73 \%$ \\
$(9.999,1.667)$ & $0.000102 V_{0}$ & $0.000103 V_{0}$ & $-0.97 \%$ \\
\hline
\end{tabular}

electric field intensity) of this area is very complicated. Since it is not easy to obtain the analytical solutions, and some simplified numerical models for electrostatic combdrives from $[4,5]$ cannot accurately simulate the fringing field, the FEM simulation [18] was used to compare with the following DBEM data. Because of the fringing of field around the edges, a large finite element model was set up in order to obtain a reasonable result. In addition, the symmetric boundary between two adjacent fingers using proper Neumann boundary conditions was used to simplify the dimensions of FEM and DBEM models.

Over 3000 points will be analyzed using coarse mesh discretization (95 elements and 95 nodes, see figure 7) of the DBEM, and compared with the reference data computed from a large refined mesh FEM model (3608 elements and 3790 nodes, see figure $8(a)$ ) because the results from the coarser mesh FEM model (1490 elements and 1607 nodes, see figure $8(b)$ ) are not adequately accurate. The results of electric potential (equipotential lines) under a refined mesh FEM and coarse mesh DBEM were shown in figure 9. Comparing the results of electric potential field using a coarse mesh DBEM and refined mesh FEM, one can see that the difference of electric potential distribution listed in table 2 is very little $(<1.3 \%)$, and the equipotential lines from coarse mesh DBEM are smoother than those from refined mesh FEM. From figure 9 , the ground plane contributes to an obvious unbalanced electrostatic field distribution if a heavily doped polysilicon film underlies the resonator and the comb structure like figure 6.

Besides the results of electric potential field, the distribution of normal electric field intensity $\left(E_{n}\right)$ on the bottom and upper sides of movable finger also needs to be studied. 


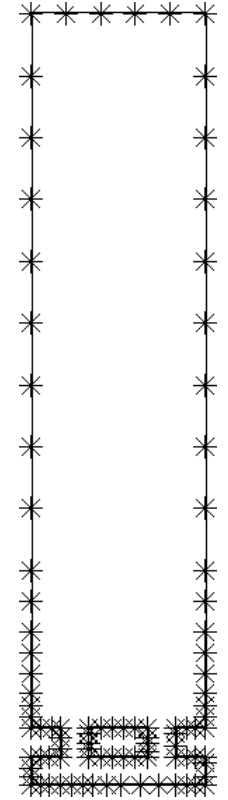

Figure 7. The related DBEM coarse mesh discretization.

Table 2. The results of electric potential of case 1 under diverse numerical methods. The origin $(x, 0,0)$ of the coordinates is located at the left bottom corner of the DBEM model shown in figure 7.

\begin{tabular}{lllr}
\hline $\begin{array}{l}\text { Locations } \\
(y, z)\end{array}$ & $\begin{array}{l}V(y, z) \text { from } \\
\text { FEM }\end{array}$ & $\begin{array}{l}V(y, z) \text { from } \\
\text { DBEM }\end{array}$ & Difference \\
\hline$(5.9867,5.0151)$ & $0.1580444 V_{p}$ & $0.15975 V_{p}$ & $+1.08 \%$ \\
$(6.1889,4.8152)$ & $0.1301514 V_{p}$ & $0.13162 V_{p}$ & $+1.13 \%$ \\
$(5.7961,4.4058)$ & $0.06673584 V_{p}$ & $0.067443 V_{p}$ & $+1.06 \%$ \\
$(1.6000,1.6000)$ & $0.0748376 V_{p}$ & $0.073904 V_{p}$ & $-1.25 \%$ \\
\hline
\end{tabular}

From figures 10 and 11, one can see that the values of normal electric field intensity $\left(E_{n, 1}\right)$ on the bottom and those $\left(E_{n, 2}\right)$ on the upper side of movable finger are both obviously dependent on the value of location on the left side of movable finger ( $\left.\operatorname{loc}_{y}\right)$. Because the charge distribution on the conductor surfaces can be determined from $\rho_{s}=\varepsilon E_{n}$ (the normal component of the electric field $E_{n}$ at a conductor boundary is equal to the surface charge density $\rho_{s}$ on the conductor divided by the permittivity $\varepsilon$ [28]) if $\varepsilon$ is a constant, the relationship between the normal force density $f_{n}$ acting on the surface of a conductor and the charge density $\rho_{s}$ of that conductor is

$$
f_{n}=-0.5 \rho_{s}^{2} / \varepsilon \text {. }
$$

Thus, the electrostatic force density $F_{n}$ acting on the movable finger along the boundary

$$
F_{n}=\int_{B} f \mathrm{~d} B
$$

can be calculated if $\rho_{s}$ (or $E_{n}$ ) is known. Therefore, the levitating force density $F$ (normal to the substrate) acting on each movable finger is equal to the difference of electrostatic force density $F_{n}$ between upper side and bottom side of concerned movable finger, and that is obviously dependent on the difference of $E_{n, 1}$ and $E_{n, 2}$. Because the difference of $E_{n, 1}$ and $E_{n, 2}$ is obvious in this case, the imbalance in the field distribution will result in a net vertical force induced on
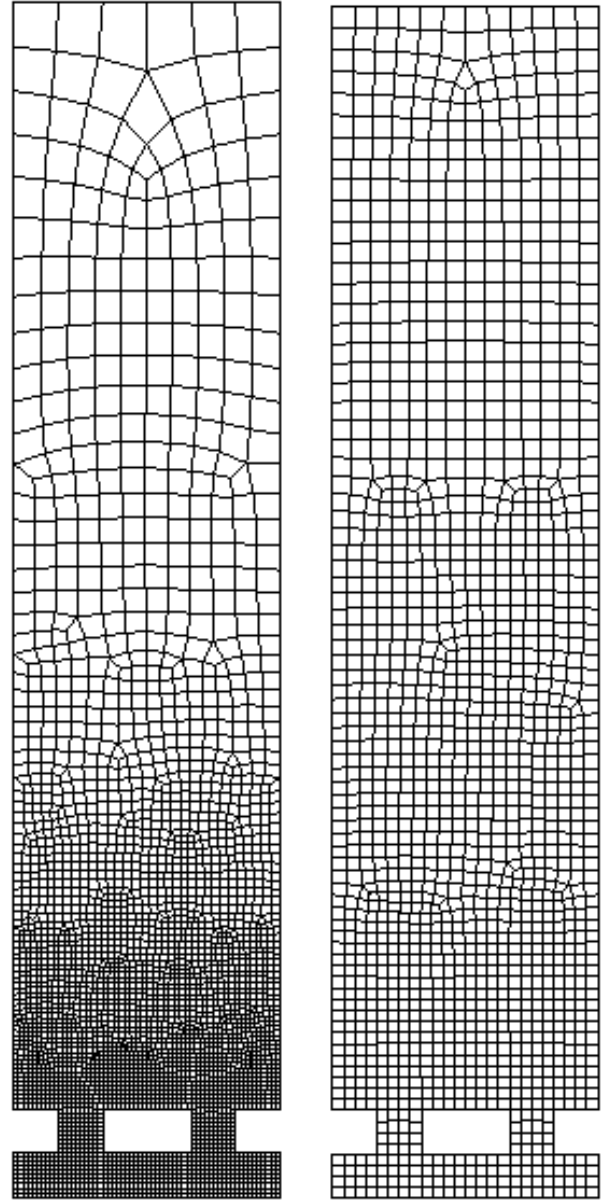

Figure 8. The related FEM mesh discretization (left part: refined mesh model; right part: coarser mesh model).

the movable comb fingers, which levitates the structure away from the substrate. Calculated by equations (21) and (22), the value of $F$ is $0.1504 \varepsilon V_{p}^{2} \mu \mathrm{m}^{-1}$.

To go a step further, the $F$ under diverse levitation $(\delta)$ and $V_{p}$ can be shown in figure 12. Some interesting results from figure 12 can be found. First, the stable equilibrium levitation, $\delta_{0},(1.19 \mu \mathrm{m}$ for this case), is the same for any nonzero bias voltages $V_{p}$. Hence, in the absence of a restoring spring force, the movable finger will be levitated to $\delta_{0}$ upon the application of a dc bias. Second, given any $\delta$, the $F$ is proportional to $V_{p}^{2}$. Because $\delta_{0}$ should be minimized for the cause of performance, the following design (cases 2 and 3) proposed by Tang et al [3] will be simulated for modification and improvement.

Besides the electric potential distribution shown in figure 9 and listed in table 2, the distribution of electric intensity field $E$ is also important. For the conventional BEM, $E$ is usually calculated by a numerical derivate of electric potential $V$ for two adjacent nodes $\left(E_{y}=-\Delta V / \Delta y\right.$ and $E_{z}=-\Delta V / \Delta z$, where $\Delta y$ and $\Delta z$ of concerned and adjacent nodes are shown in figure 13), and $E$ can be obtained by direct calculation using the hypersingular formulation of the DBEM. For $V_{p}$, the results of electric field intensity $E_{y}$ and $E_{z}$ of case 1 under diverse values of $\Delta y$ and $\Delta z$ by way of the BEM and DBEM are listed in tables 3 and 4 , and the differences of $E_{y}$ and $E_{z}$ in this case between the BEM and DBEM are shown in figure 14. By way of the conventional BEM, figure 14 shows 

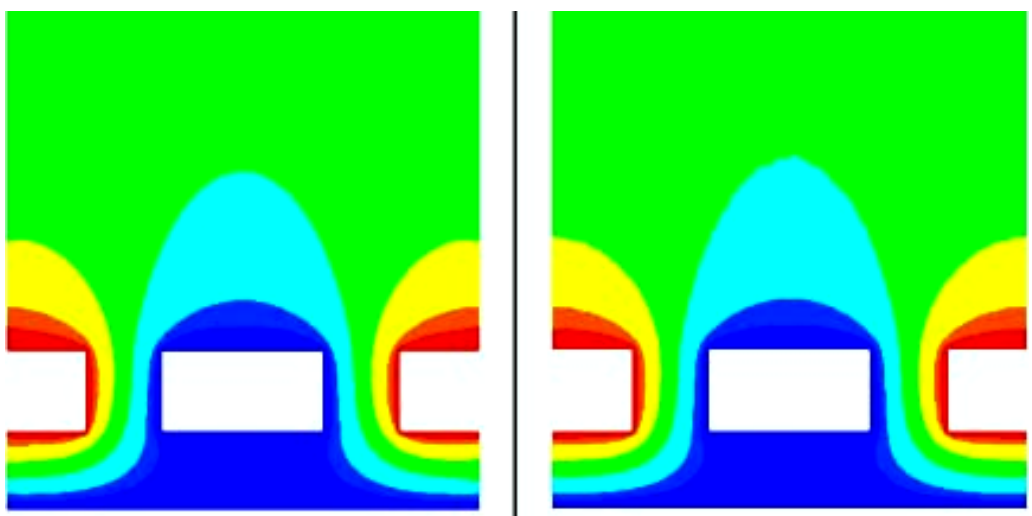

Figure 9. Results of electric potential field (equipotential lines) of case 1 using the coarse mesh DBEM (left part) and refined mesh FEM (right part).

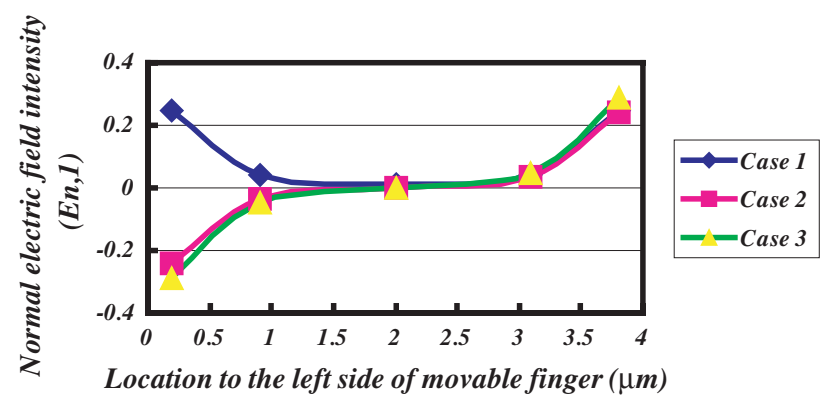

Figure 10. The distribution of normal electric field intensity $E_{n, 1}$ on the bottom of the movable finger under diverse design cases (unit: $V_{p} \mu \mathrm{m}^{-1}$ ).

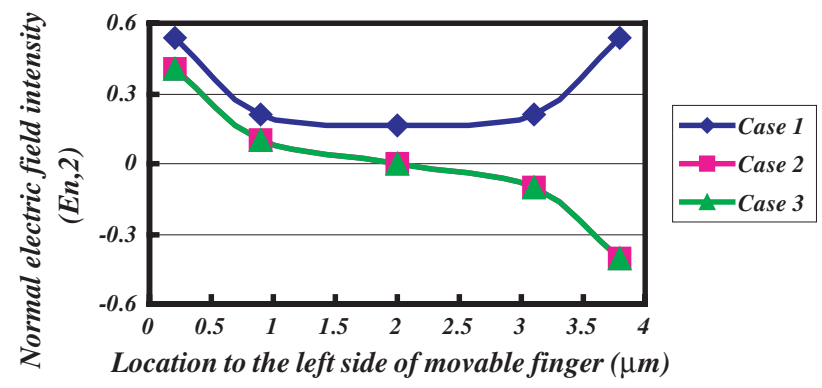

Figure 11. The distribution of normal electric field intensity $\left(E_{n, 2}\right)$ on the upper side of the movable finger under diverse design cases (unit: $V_{p} \mu \mathrm{m}^{-1}$ )

that the larger the $\Delta y$ and $\Delta z$ are, the less the accuracy is, and the accuracy of $E_{z}$ is better than $E_{y}$ for the same value of $\Delta y$ and $\Delta z$. One can also see that we need more auxiliary nodes than DBEM to calculate the derivate of $V$ of two adjacent nodes for solving the $E$ of each concerned node if using the conventional BEM. Therefore, the accuracy and performance of the DBEM are better than those of the conventional BEM for case 1 .

Case 2. Although the electric field distribution becomes balanced if the ground plane is eliminated and the substrate beneath the structures is removed, or a top ground plane above the combdrive is suspended, it is impracticable and infeasible for MEMS devices because both of these approaches require much more complex manufacturing processes. There

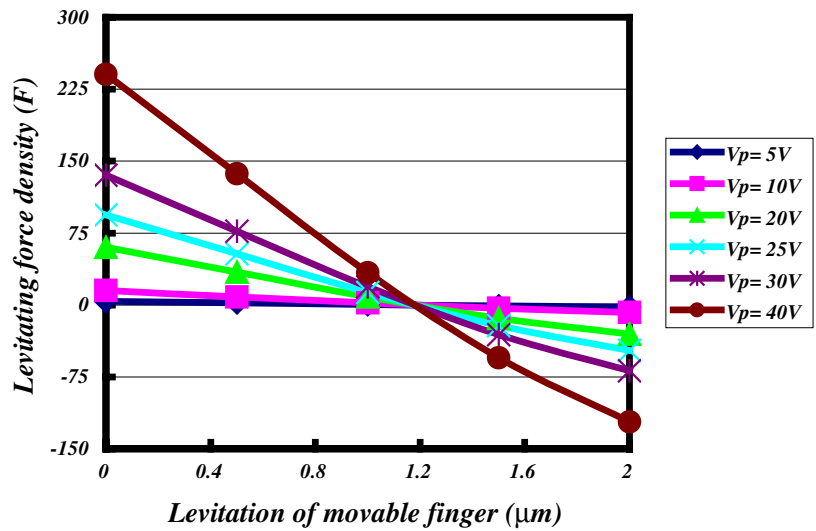

Figure 12. The levitating force density $(F)$ acting on the movable finger under diverse levitation and $V_{p}$ (unit: $\varepsilon V^{2} \mu \mathrm{m}^{-1}$ ).

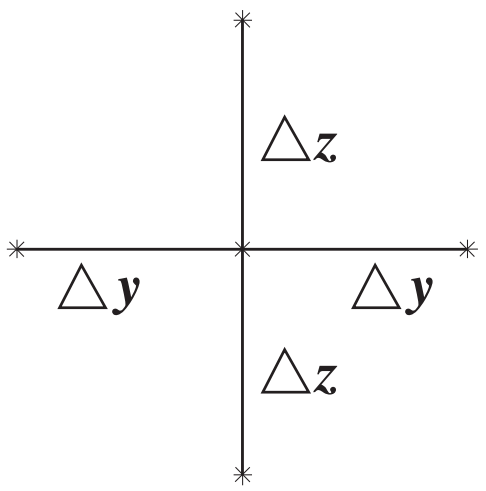

Figure 13. The relative locations of concerned and adjacent nodes.

are two simple and efficient control methods proposed by Tang et al [3] to reduce the levitating force. A simpler solution is to modify the combdrive itself, which is to reverse the polarity on alternating drive fingers results in the field distribution shown in figure 15 . For a grounded movable comb finger, a differential dc bias $V_{p}$ is applied to the two adjacent electrodes shown in figure 15 . Let us determine the electric field distribution.

The results of electric potential under the refined mesh FEM and coarse mesh DBEM are shown in figure 16, and one can see that the difference of electric potential listed in 


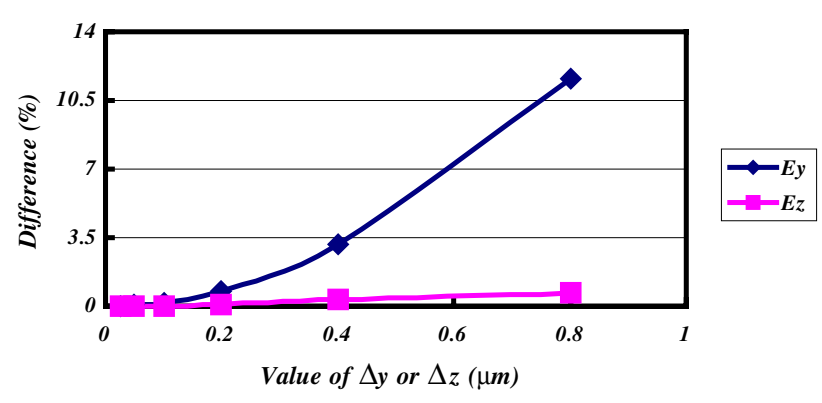

Figure 14. The differences of $E_{y}$ and $E_{z}$ of case 1 between the BEM and DBEM.

Table 3. The results of electric field intensity $E_{y}$ of case 1 under diverse values of $\Delta y$ by way of the BEM and DBEM.

\begin{tabular}{lllr}
\hline $\begin{array}{l}\text { Location of } \\
\text { concerned node } \\
(x, 5.0,5.0)\end{array}$ & $\begin{array}{l}E_{y}(y, z) \text { from } \\
\text { BEM }\end{array}$ & $\begin{array}{l}E_{y}(y, z) \text { from } \\
\text { DBEM }\end{array}$ & Difference \\
\hline$\Delta y=0.025 \mu \mathrm{m}$ & $0.055301871 V_{p}$ & & $+0.020 \%$ \\
$\Delta y=0.05 \mu \mathrm{m}$ & $0.055316325 V_{p}$ & & $+0.046 \%$ \\
$\Delta y=0.10 \mu \mathrm{m}$ & $0.055401526 V_{p}$ & $0.055291 V_{p}$ & $+0.200 \%$ \\
$\Delta y=0.20 \mu \mathrm{m}$ & $0.05573304 V_{p}$ & & $+0.799 \%$ \\
$\Delta y=0.40 \mu \mathrm{m}$ & $0.057026338 V_{p}$ & & $+3.139 \%$ \\
$\Delta y=0.80 \mu \mathrm{m}$ & $0.06172560 V_{p}$ & & $+11.638 \%$ \\
\hline
\end{tabular}

Table 4. The results of electric field intensity $E_{z}$ of case 1 under diverse values of $\Delta z$ by way of the BEM and DBEM.

\begin{tabular}{llll}
\hline $\begin{array}{l}\text { Location of } \\
\text { concerned node } \\
(x, 5.0,5.0)\end{array}$ & $E_{z}(y, z)$ from & $\begin{array}{l}E_{z}(y, z) \text { from } \\
\text { DEM }\end{array}$ & \\
\hline$\Delta z=0.025 \mu \mathrm{m}$ & $-0.1487479 V_{p}$ & & Difference \\
$\Delta z=0.05 \mu \mathrm{m}$ & $-0.1487582 V_{p}$ & & $-0.001 \%$ \\
$\Delta z=0.10 \mu \mathrm{m}$ & $-0.1487879 V_{p}$ & $-0.14875 V_{p}$ & $+0.006 \%$ \\
$\Delta z=0.20 \mu \mathrm{m}$ & $-0.1488855 V_{p}$ & & $+0.025 \%$ \\
$\Delta z=0.40 \mu \mathrm{m}$ & $-0.1492378 V_{p}$ & & $+0.091 \%$ \\
$\Delta z=0.80 \mu \mathrm{m}$ & $-0.1497661 V_{p}$ & & $+0.683 \%$ \\
\hline
\end{tabular}

table 5 is small (3\%). From figure 16, vertical electric fields terminating on the top surface of the movable comb finger are eliminated because the electric potential gradient of the top surface of the central movable comb finger is very small, and the $F$ can be brought down successfully. From figures 10 and 11 , one can also find that the values of $E_{n, 1}$ and $E_{n, 2}$ are both obviously dependent on the value of $\operatorname{loc}_{y}$, but the $F$ is almost zero because of the force balance in this case. Therefore, we can conclude that the design of case 2 can reduce the levitation very efficiently.

Besides the electric potential distribution shown in figure 16 and listed in table 5, the results of electric field intensity $E_{y}$ and $E_{z}$ of case 2 under diverse values of $\Delta y$

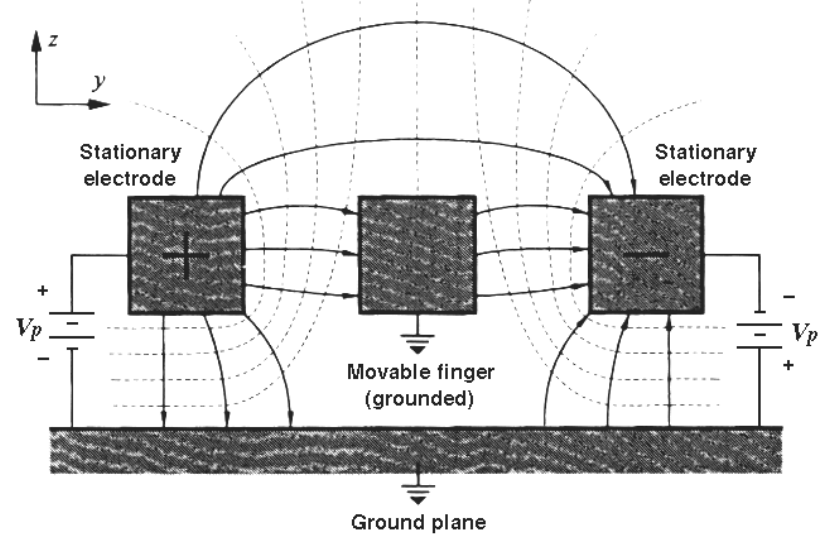

Figure 15. Cross section of the potential contours (dashed lines) and the electric fields (solid lines) around a movable comb finger when differential dc bias is applied to the two adjacent electrodes.

Table 6. The results of electric field intensity $E_{y}$ of case 2 under diverse values of $\Delta y$ by way of the BEM and DBEM.

\begin{tabular}{llll}
\hline $\begin{array}{l}\text { Location of } \\
\text { concerned node } \\
(x, 5.0,5.0)\end{array}$ & $\begin{array}{l}E_{y}(y, z) \text { from } \\
\text { BEM }\end{array}$ & $\begin{array}{l}E_{y}(y, z) \text { from } \\
\text { DBEM }\end{array}$ & Difference \\
\hline$\Delta y=0.025 \mu \mathrm{m}$ & $0.083717532 V_{p}$ & & $+0.003 \%$ \\
$\Delta y=0.05 \mu \mathrm{m}$ & $0.083747707 V_{p}$ & & $+0.039 \%$ \\
$\Delta y=0.10 \mu \mathrm{m}$ & $0.08343194 V_{p}$ & $0.083715 V_{p}$ & $+0.150 \%$ \\
$\Delta y=0.20 \mu \mathrm{m}$ & $0.084224291 V_{p}$ & & $+0.610 \%$ \\
$\Delta y=0.40 \mu \mathrm{m}$ & $0.08572723 V_{p}$ & & $+2.400 \%$ \\
$\Delta y=0.80 \mu \mathrm{m}$ & $0.091383345 V_{p}$ & & $+9.160 \%$ \\
\hline
\end{tabular}

and $\Delta z$ by way of BEM and DBEM are also listed in tables 6 and 7, and the differences of $E_{y}$ and $E_{z}$ between BEM and DBEM are shown in figure 17. By way of conventional BEM, figure 17 also shows that the larger the $\Delta y$ and $\Delta z$ are, the less the accuracy is, and the accuracy of $E_{z}$ is also better than $E_{y}$ for the same value of $\Delta y$ and $\Delta z$. We also can find that the accuracy and performance of the DBEM are better than those of the conventional BEM for this case.

Case 3. To further suppress levitation, the ground plane is modified such that underneath each comb finger there is a strip of conductor biased at the same potential, as illustrated in figure 18. For a grounded movable comb finger, a differential dc bias $V_{p}$ is applied to the two adjacent electrodes and the striped ground conductors shown in figure 18. Let us determine the electric field distribution.

The results of electric potential under refined mesh FEM and coarse mesh DBEM are shown in figure 19, and the difference listed in table 8 is also very small $(<1.7 \%)$. In this case, the polysilicon layer is used to form the crossovers to electrically isolate alternating comb fingers, and figure 19

Table 5. The results of electric potential of case 2 under diverse numerical methods. The origin $(x, 0,0)$ of the coordinates is located at the left bottom corner of the DBEM model shown in figure 7.

\begin{tabular}{llll}
\hline $\begin{array}{l}\text { Locations } \\
(y, z)\end{array}$ & $\begin{array}{l}V(y, z) \text { from } \\
\text { FEM }\end{array}$ & $\begin{array}{l}V(y, z) \text { from } \\
\text { DBEM }\end{array}$ & Difference \\
\hline$(6.1889,4.8152)$ & $-9.55811 \times 10^{-3} V_{p}$ & $-9.8519 \times 10^{-3} V_{p}$ & $+3.07 \%$ \\
$(5.7961,4.4058)$ & $5.5481 \times 10^{-3} V_{p}$ & $5.589 \times 10^{-3} V_{p}$ & $+0.74 \%$ \\
$(1.6000,1.6000)$ & $7.48254 \times 10^{-1} V_{p}$ & $7.389 \times 10^{-1} V_{p}$ & $-1.25 \%$ \\
\hline
\end{tabular}



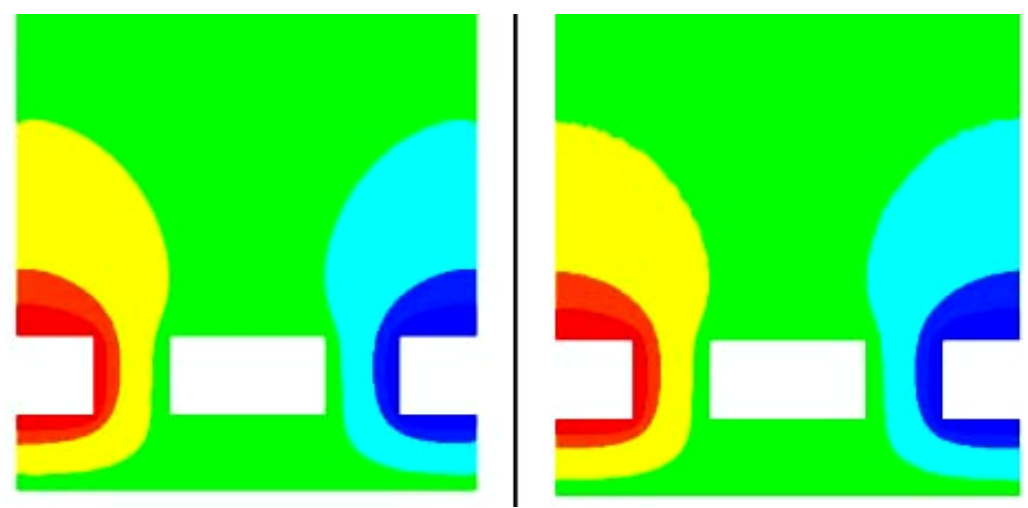

Figure 16. Results of electric potential field (equipotential lines) of case 2 using the coarse mesh DBEM (left part) and refined mesh FEM (right part).
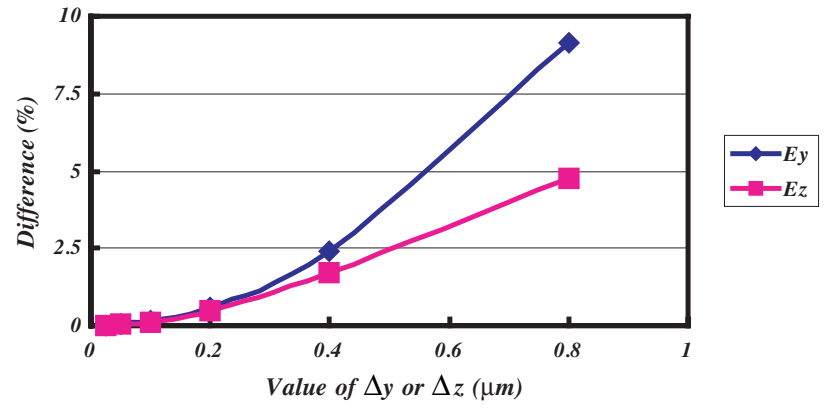

Figure 17. The differences of $E_{y}$ and $E_{z}$ of case 2 between the BEM and DBEM.

Table 7. The results of electric field intensity $E_{z}$ of case 2 under diverse values of $\Delta z$ by way of the BEM and DBEM.

\begin{tabular}{llll}
\hline $\begin{array}{l}\text { Location of } \\
\text { concerned node } \\
(x, 5.0,5.0)\end{array}$ & $\begin{array}{l}E_{z}(y, z) \text { from } \\
\text { BEM }\end{array}$ & $\begin{array}{l}E_{z}(y, z) \text { from } \\
\text { DBEM }\end{array}$ & Difference \\
\hline$\Delta z=0.025 \mu \mathrm{m}$ & $-0.04184856 V_{p}$ & & $+0.016 \%$ \\
$\Delta z=0.05 \mu \mathrm{m}$ & $-0.04185452 V_{p}$ & & $+0.030 \%$ \\
$\Delta z=0.10 \mu \mathrm{m}$ & $-0.04188907 V_{p}$ & $-0.041842 V_{p}$ & $+0.110 \%$ \\
$\Delta z=0.20 \mu \mathrm{m}$ & $-0.04203393 V_{p}$ & & $+0.460 \%$ \\
$\Delta z=0.40 \mu \mathrm{m}$ & $-0.04256453 V_{p}$ & & $+1.730 \%$ \\
$\Delta z=0.80 \mu \mathrm{m}$ & $-0.04383965 V_{p}$ & $+4.770 \%$ \\
\hline
\end{tabular}

shows that the $F$ can be decreased enormously because the electric potential gradient of the top surface of the central movable comb finger is almost zero. From figures 10 and 11 , we can also find that the values of $E_{n, 1}$ and $E_{n, 2}$ are both obviously dependent on the value of $\operatorname{loc}_{y}$, but the value of $F$ is almost zero because of a force balance like case 2 . Therefore, this design can also efficiently reduce the levitating force. In addition, experimental data from [3] even show that the levitating force is suppressed by over an order of magnitude compared to the original biasing scheme shown in figure 6 .

Besides the electric potential distribution shown in figure 19 and listed in table 8, the results of electric field intensity $E_{y}$ and $E_{z}$ in this case under diverse values of $\Delta y$ and $\Delta z$ by way of the BEM and DBEM are also listed in tables 9 and 10 , and the differences of $E_{y}$ and $E_{z}$ between the BEM and DBEM are shown in figure 20. From the results shown

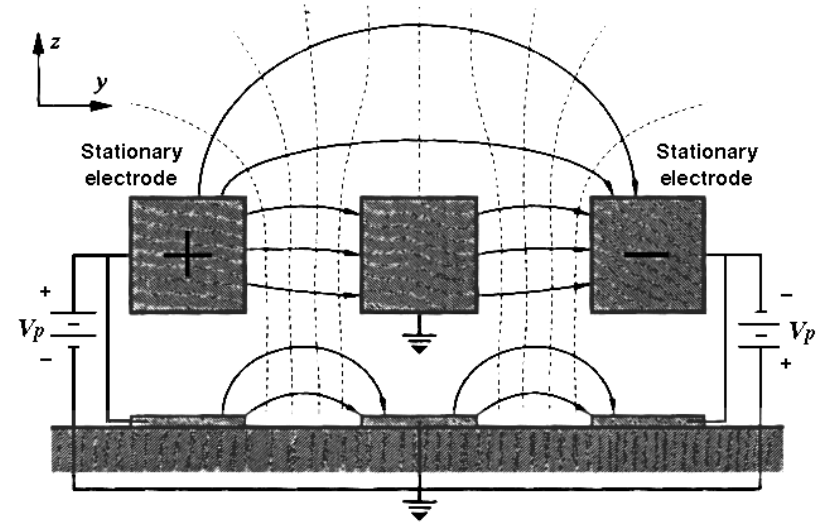

Figure 18. Cross section of the potential contours (dashed lines) and the electric fields (solid lines) around a movable comb finger when differential dc bias is applied to the two adjacent electrodes and the striped ground conductors.

in figure 20, we can understand that the larger the $\Delta y$ and $\Delta z$ are, the less the accuracy is, and the accuracy of $E_{z}$ is also better than $E_{y}$ for the same value of $\Delta y$ and $\Delta z$ if using the conventional BEM. Hence, one can also be convinced that the DBEM is an efficient method for solving the electrostatic problem of MEMS combdrive.

\section{Discussions}

(1) Because levitating force control can get a higher quality factor of a combdrive, how to get an accurate electrostatic field is very important and indispensable for the design of MEMS devices. Though some simplified numerical models for electrostatic combdrives can be found in $[4,5]$, there are three types of fringing fields not taken into account, which result from the ground plane, widths and heights of fixed and movable fingers. After using a coarse mesh DBEM to accurately calculate the electrostatic response of the comb finger biased with a dc voltage, the induced vertical force per unit length of the movable comb finger at different levitation positions can be obtained. Then this vertical force density can be plotted against levitation at different dc bias voltages, as in [4, 5]. Besides the levitating force case, driving force control can 
Table 8. The results of electric potential of case 3 under diverse numerical methods. The origin $(x, 0,0)$ of the coordinates is located at the left bottom corner of DBEM model shown in figure 7.

\begin{tabular}{llrl}
\hline $\begin{array}{l}\text { Locations } \\
(y, z)\end{array}$ & \multicolumn{1}{l}{$\begin{array}{l}V(y, z) \text { from } \\
\text { FEM }\end{array}$} & \multicolumn{1}{l}{$\begin{array}{l}V(y, z) \text { from } \\
\text { DBEM }\end{array}$} & Difference \\
\hline$(6.1889,4.8152)$ & $-9.55871 \times 10^{-3} V_{p}$ & $-9.5503 \times 10^{-3} V_{p}$ & $-0.09 \%$ \\
$(5.7961,4.4058)$ & $5.57861 \times 10^{-3} V_{p}$ & $5.5616 \times 10^{-3} V_{p}$ & $-0.30 \%$ \\
$(1.6000,1.6000)$ & $8.91016 \times 10^{-1} V_{p}$ & $8.7603 \times 10^{-1} V_{p}$ & $-1.68 \%$ \\
\hline
\end{tabular}
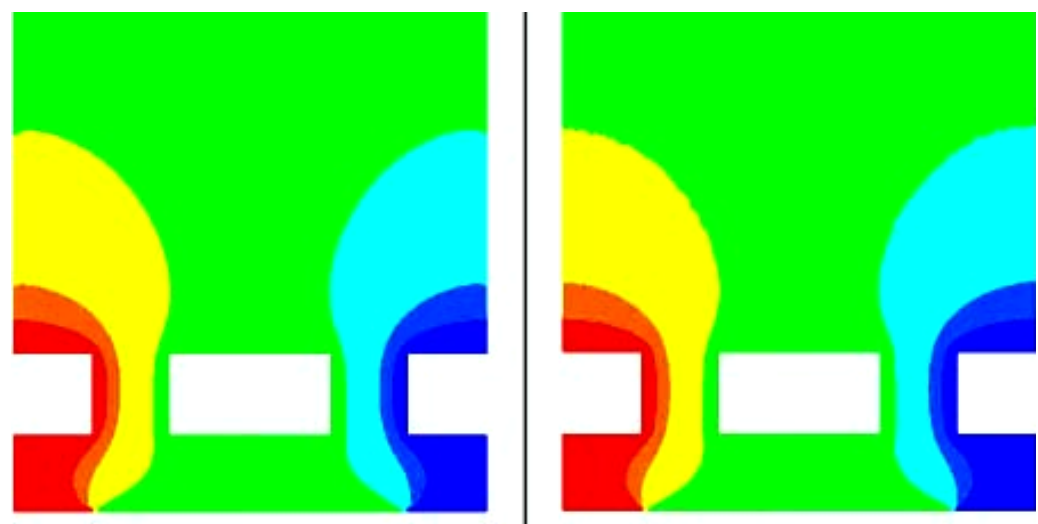

Figure 19. Results of electric potential field (equipotential lines) of case 3 using the coarse mesh DBEM (left part) and refined mesh FEM (right part).

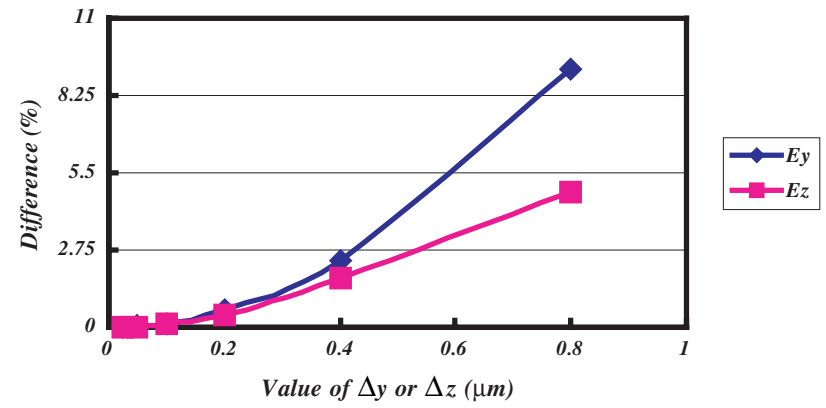

Figure 20. The differences of $E_{y}$ and $E_{z}$ of case 3 between the BEM and DBEM.

also utilize the coarse mesh DBEM to deal with related electrostatic problems.

(2) Results show that the values of normal electric field intensity $\left(E_{n, 1}\right)$ on the bottom and those $\left(E_{n, 2}\right)$ on the upper side of a movable finger are both obviously dependent on the location on the left side of movable finger $\left(\operatorname{loc}_{y}\right)$ under diverse design cases. Because the difference of $E_{n, 1}$ and $E_{n, 2}$ is obvious in case 1, the imbalance in the field distribution will result in a net vertical force induced on the movable comb fingers, which levitates the structure away from the substrate, but the values of levitating force density $(F)$ of cases 2 and 3 are almost zero because of the force and moment balance for the movable finger. Therefore, we can conclude that cases 2 and 3 can efficiently suppress the levitation of a MEMS combdrive because the levitating force density of the movable finger concerned can be reduced from 0.1504 $\varepsilon V_{p}^{2} \mu \mathrm{m}^{-1}$ to zero.

(3) Results also show that the stable equilibrium levitation, $\delta_{0},(1.19 \mu \mathrm{m}$ for case 1$)$, is the same for any nonzero bias
Table 9. The results of electric field intensity $E_{y}$ of case 3 under diverse values of $\Delta y$ by way of the BEM and DBEM.

\begin{tabular}{lllc}
\hline $\begin{array}{l}\text { Location of } \\
\text { concerned node } \\
(x, 5.0,5.0)\end{array}$ & $\begin{array}{l}E_{y}(y, z) \text { from } \\
\text { BEM }\end{array}$ & $\begin{array}{l}E_{y}(y, z) \text { from } \\
\text { DBEM }\end{array}$ & Difference \\
\hline$\Delta y=0.025 \mu \mathrm{m}$ & $0.083906777 V_{p}$ & & $+0.012 \%$ \\
$\Delta y=0.05 \mu \mathrm{m}$ & $0.083926298 V_{p}$ & & $+0.035 \%$ \\
$\Delta y=0.10 \mu \mathrm{m}$ & $0.084025361 V_{p}$ & $0.083897 V_{p}$ & $+0.153 \%$ \\
$\Delta y=0.20 \mu \mathrm{m}$ & $0.084407292 V_{p}$ & & $+0.608 \%$ \\
$\Delta y=0.40 \mu \mathrm{m}$ & $0.085915655 V_{p}$ & & $+2.406 \%$ \\
$\Delta y=0.80 \mu \mathrm{m}$ & $0.091586903 V_{p}$ & $+9.170 \%$ \\
\hline
\end{tabular}

Table 10. The results of electric field intensity $E_{z}$ of case 3 under diverse values of $\Delta z$ by way of the BEM and DBEM.

\begin{tabular}{llll}
\hline $\begin{array}{l}\text { Location of } \\
\text { concerned node } \\
(x, 5.0,5.0)\end{array}$ & $\begin{array}{l}E_{z}(y, z) \text { from } \\
\text { BEM }\end{array}$ & $\begin{array}{l}E_{z}(y, z) \text { from } \\
\text { DBEM }\end{array}$ & Difference \\
\hline$\Delta z=0.025 \mu \mathrm{m}$ & $-0.04179000 V_{p}$ & & $-0.004 \%$ \\
$\Delta z=0.05 \mu \mathrm{m}$ & $-0.04180423 V_{p}$ & & $+0.030 \%$ \\
$\Delta z=0.10 \mu \mathrm{m}$ & $-0.04183837 V_{p}$ & $-0.041792 V_{p}$ & $+0.112 \%$ \\
$\Delta z=0.20 \mu \mathrm{m}$ & $-0.04198500 V_{p}$ & & $+0.462 \%$ \\
$\Delta z=0.40 \mu \mathrm{m}$ & $-0.04251922 V_{p}$ & $+1.741 \%$ \\
$\Delta z=0.80 \mu \mathrm{m}$ & $-0.04380557 V_{p}$ & & $+4.820 \%$ \\
\hline
\end{tabular}

voltages $V_{p}$. Hence, in the absence of a restoring spring force, the movable finger will be levitated to $\delta_{0}$ upon the application of a dc bias. Second, given any levitation $(\delta)$, the $F$ is proportional to the square of the applied dc bias, $V_{p}^{2}$.

(4) By comparing the element mesh of the refined mesh FEM and coarse mesh DBEM of an electrostatic combdrive, considering the fringing of field around the edges, one can see that the numbers of elements and nodes for the refined mesh FEM are much higher than those of the coarse mesh 
DBEM to get a reasonable result. Though using the FEM is widespread for MEMS devices nowadays, it is still very difficult to establish the boundary conditions and generate all the proper FEM because the values of gap for MEMS combdrives_-gaps between fingers and from fingers to the ground plane-always change many times before final layout in the variable design stage. Therefore, we strongly recommend the DBEM for studying the electrostatic field of the levitation of MEMS combdrives because the DBEM's discretizations are restricted only to the boundaries, and it makes data generation much easier than FEM.

\section{Conclusions}

The dual integral formulation of electrostatic combdrive problems, considering the fringing of field around the edges, has been presented in this paper. Comparisons of the results between the FEM and DBEM analyses were discussed with respect to diverse design cases for electrostatic MEMS combdrives in order to demonstrate the efficiency of the DBEM. It has been ensured that the capabilities of coarse mesh DBEM simulation are acceptable after comparison with the refined mesh FEM data. For electrical engineering practices, since the numbers of elements and nodes for the refined mesh FEM are much higher than those of the coarse mesh DBEM to get a reasonable result, and it wastes much time for diverse values of gap design if using the domain-type FEM, so the present boundary-type DBEM has great potential for industrial applications, especially in the initial variable design stage.

\section{Acknowledgments}

The authors are particularly grateful for the valuable comments from the reviewers and editorial board member of JMM.

\section{References}

[1] Tang W C, Nguyen T C H, Judy M W and Howe R T 1989 Lateral driven polysilicon resonant microstructures Sensors Actuators 20 25-32

[2] Tang W C, Nguyen T C H, Judy M W and Howe R T 1990 Electrostatic-comb drive of lateral polysilicon resonators Sensors Actuators A 21-23 328-31

[3] Tang W C, Lim M G and Howe R T 1992 Electrostatic comb drive levitation and control method IEEE J. Microelectromech. Syst. 1 170-8

[4] Yeh J L A, Hui C Y and Tien N C 2000 Electrostatic model for an asymmetric combdrive IEEE J. Microelectromech. Syst. 9 126-35

[5] Johnson W A and Warne L K 1995 Electrophysics of micromechanical comb actuators IEEE J. Microelectromech. Syst. 4 49-59

[6] Ye W, Mukherjee S and MacDonald N C 1998 Optimal shape design of an electrostatic comb drive in microelectromechanical systems IEEE $J$. Microelectromech. Syst. 7 16-26

[7] Sadiku M N O 1992 Numerical Techniques in Electromagnetics (Boca Raton, FL: CRC Press)
[8] Beerschwinger U, Miline N G, Yang S J, Reuben R L, Sangster A J and Ziad H 1994 Coupled electrostatic and mechanical FEA of a micromotor IEEE J. Microelectromech. Syst. 3 162-71

[9] Jin J 2002 The Finite Element Method in Electromagnetics (New York: Wiely)

[10] Aliabadi M H 2002 The Boundary Element Method (New York: Wiely)

[11] Senturia S D, Aluru N and White J 1997 Simulating the behavior of MEMS devices: computational methods and needs IEEE Comput. Sci. Eng. Mag. January-March $30-43$

[12] Nabors K and White J K 1992 Multipole-accelerated capacitance extraction algorithrms for 3-D structures with multiple dielectrics IEEE Trans. Circuits Syst. 39 946-54

[13] Yu W, Wang Z and Gu J 2003 Fast capacitance extraction of actual 3-D VLSI interconnects using quasi-multiple medium accelerated BEM IEEE Trans. Microw. Theory Tech. 51 109-19

[14] Phillips J R and White J K 1997 A precorrected-FFT method for electrostatic analysis of complicated 3-D structures IEEE Trans. Computer-Aided Design 16 1059-72

[15] Leung C Y and Walker S P 1997 Iterative solution of large three-dimensional BEM elastostatic analysis using the GMRES technique Int. J. Numer. Methods Eng. 40 2227-36

[16] Chen J T and Chen K H 2004 Applications of the dual integral formulation in conjunction with fast multipole method in large-scale problems for 2D exterior acoustics Eng. Anal. Bound. Elem. 28 687-709

[17] Chew W C, Chao H Y, Cui T J, Lu C C, Ohnuki S, Pan Y C, Song J M, Velamparambil S and Zhao J S 2003 Fast integral equation solvers in computational electromagnetics of complex structures Eng. Anal. Bound. Elem. 27 803-23

[18] I-DEAS User's Guide 1990 Finite Element Modeling SDRC

[19] Chen J T and Hong H K 1999 Review of dual boundary element methods with emphasis on hypersingular integrals and divergent series Trans. ASME, Appl. Mech. Rev. 52 $17-33$

[20] Chyuan S W, Liao Y S and Chen J T 2003 An efficient method for solving electrostatic problems IEEE Comput. Sci. Eng. Mag. May-June 52-8

[21] Christiansen S 2001 Detecting non-uniqueness of solutions to biharmonic integral equations through SVD J. Comput. Appl. Math. 134 23-35

[22] Harrington R F, Pontoppidan K, Abrahamsen P and Albertsen N C 1969 Computation of Laplacian potentials by an equivalent-source method Proc. IEE $\mathbf{1 1 6}$ 1715-20

[23] Chen J T, Lin J H, Kuo S R and Chiu Y P 2001 Analytical study and numerical experiments for degenerate scale problems in boundary element method using degenerate kernels and circulants Eng. Anal. Bound. Elem. 25 819-28

[24] Chen J T and Lin S R 2002 On the rank-deficiency problems in boundary integral formulation using the Fredholm alternative theory and singular value decomposition technique Proc. 5th World Congress on Computational Mechanics (Vienna, Austria, 7-12 July)

[25] Chen J T and Hong H K 1994 Dual boundary integral equations at a corner using contour approach around singularities Adv. Eng. Software 21 169-78

[26] Huber O, Lang A and Kuhn G 1993 Evaluation of the stress tensor in 3D elastostatics by direct solving of hypersingular integrals Comput. Mech. 12 39-50

[27] Liang M T, Chen J T and Yang S S 1999 Error estimation for boundary element method Eng. Anal. Bound. Elem. 23 257-65

[28] Cheng D K 1989 Field and Wave Elctromagnetics (Reading, NA: Addison-Wesley) 